\title{
Síndrome de Cushing Endógena: Características Clínico- Laboratoriais em 73 Casos
}

\section{artigo original}

\author{
LUCIO VILAR \\ LUCIANA A. NAVES \\ Maria da ConceiçÃo Freitas \\ ELIANE MOURA \\ VIVIANE CANADAS \\ EDMUNDO LEAL \\ LUCIANO TEIXEIRA \\ AMARO GUSMÃO \\ VERA SANTOS \\ RenATA CAMPOS \\ MARISE LIMA \\ RenATA PAIVA \\ José LUCIANo AlbuQUerQUe \\ CÉlIA S. EgITO \\ Carlos A. Botelho \\ RUY LYRA \\ Monalisa F. AZEVEDo \\ LuIz Augusto CAsulari
}

Serviço de Endocrinologia do

Hospital das Clínicas da UFPE

(LV, EM, VC, EL, LT, AG, VS, RC,

$M L, R P, J L A, C S E$ \& CAB), Centro

de Diabetes e Endocrinologia

de Pernambuco (LV, AG \& MCF)

e Instituto de Endocrinologia do

Recife (RL), PE; e Serviço de

Endocrinologia do Hospital

Universitário de Brasilia (MFA,

LAC \& LAN), Brasilia, DF.

Recebido em 30/08/06

Revisado em 23/1 1/06

Aceito em 04/12/06

\section{RESUMO}

Avaliamos as características clínico-laboratoriais de 73 pacientes com síndrome de Cushing (SC) endógena, assim distribuídos: 46 (63\%) com doença de Cushing (DC), $21(28,7 \%)$ com tumores adrenais (TA) e $6(8,2 \%)$ com a síndrome do ACTH ectópico (SAE). A freqüência de manifestações clássicas do hipercortisolismo foi similar, independentemente da etiologia da SC. Em 100\% dos casos de SC, observaram-se níveis do cortisol sérico (CS) $>1,8 \mu \mathrm{g} / \mathrm{dL}$ após supressão com doses baixas de dexametasona (DMS), além de elevação do cortisol à meia-noite (sérico ou salivar). Contudo, o cortisol livre urinário foi normal em $11,5 \%$ dos pacientes. Os níveis de ACTH mostraram-se suprimidos nos pacientes com TA, normais ou elevados na DC e sempre elevados na SAE. No teste de supressão noturna com $8 \mathrm{mg}$ de DMS, supressão do CS $>50 \%$ foi observada em $78,2 \%$ dos casos de $\mathrm{DC}$ e $33,3 \%$ dos casos de $\mathrm{SAE}$, enquanto uma supressão $>80 \%$ foi exclusiva da DC. Após estímulo com CRH ou DDAVP, um incremento do ACTH $\geq 35 \%$ aconteceu em $81 \%$ dos indivíduos com DC e em $16,6 \%$ daqueles com $\mathrm{SAE}$, ao passo que um incremento do ACTH $>50 \%$ restringiu-se à DC. A combinação de incremento do $\mathrm{ACTH} \geq 35$ e supressão do CS $>50 \%$ foi também exclusiva da DC. A ressonância magnética visualizou $100 \%$ dos macroadenomas e $59,4 \%$ dos microadenomas hipofisários nos casos de DC. Em 10 pacientes submetidos ao cateterismo bilateral do seio petroso inferior, um gradiente centro-periferia de $\mathrm{ACTH} \geq 3$ pós-CRH ou DDAVP teve sensibilidade de $90 \%$ e especificidade de $100 \%$ para a doença de Cushing. (Arq Bras Endocrinol Metab 2007;51/4:566-574)

Descritores: Síndrome de Cushing; Hipercortisolismo; Doença de Cushing; Cortisol

\section{ABSTRACT}

Endogenous Cushing's Syndrome: Clinical and Laboratorial Features in 73 Cases.

We studied clinical and laboratorial features of 73 patients with endogenous Cushing's syndrome, subdivided as follows: 46 (63\%) with Cushing's disease (CD), $21(28.7 \%)$ with an adrenal tumor and $6(8.2 \%)$ with ectopic ACTH secretion (EAS). The rate of typical manifestations of hypercortisolism was similar regardless its etiology. In 100\% of cases of Cushing's syndrome we observed serum cortisol levels greater than $1.8 \mu \mathrm{g} / \mathrm{dL}$ in low-dose dexamethasone (DMS) suppression tests, as well as elevation of serum or salivary midnight cortisol. However, urinary free cortisol was normal in $11.5 \%$ of patients. ACTH levels were suppressed in patients with adrenal tumors, normal or high in CD and always high in EAS. In the 8-mg overnight DMS suppression test, serum cortisol suppression $>50 \%$ was observed in $78.2 \%$ of cases of CD and in $33.3 \%$ of subjects with EAS, while an $80 \%$ suppression was only seen in CD. After stimulation with $\mathrm{CRH}$ or DDAVP an ACTH increase $\geq 35 \%$ occurred in $81 \%$ of individuals with $C D$ and $16.6 \%$ of those with EAS, while an ACTH increase $\geq 50$ achieved $100 \%$ specificity. Moreover, the combination of serum cortisol suppression $>50 \%$ and an ACTH increase $\geq 35 \%$ in both tests only occurred in Cushing's disease. Pituitary magnetic resonance imaging identified $100 \%$ of macroadenomas and $59.4 \%$ of microadenomas in patients with CD. Among 10 patients that underwent bilateral inferior petrosal sinus sampling, a central-toperipheral ACTH gradient $\geq 3$ after $\mathrm{CRH}$ or DDAVP had $90 \%$ sensitivity and $100 \%$ specificity for Cushing's disease. (Arq Bras Endocrinol Metab 2007;51/4:566-574)

Keywords: Cushing's syndrome; Hypercortisolism; Cushing's disease, Cortisol 
Escosing NTRE OS DISTÚRBIOS ENDÓCRINOS, a síndrome de Cushing (SC) certamente é um dos que mais fascinam clínicos e endocrinologistas pelas dificuldades que muitas vezes surgem para seu diagnóstico e determinação de sua etiologia. O diagnóstico da SC envolve a confirmação do hipercortisolismo e a investigação do seu fator causal. Neste contexto, é fundamental a exclusão da SC decorrente do uso de glicocorticóides, incluindo preparações tópicas, colírios e gotas nasais, freqüentemente omitidas pelos pacientes (1). Classicamente, os adenomas hipofisários secretores de ACTH (doença de Cushing) são a causa mais comum da SC endógena (cerca de 70\% dos casos), seguida das patologias adrenais (tumores ou, mais raramente, hiperplasia adrenal bilateral) e a síndrome do ACTH ectópico (SAE) $(2,3)$.

Para a confirmação do hipercortisolismo, os exames mais utilizados são os testes de supressão com doses baixas de dexametasona (LDDST), a dosagem do cortisol livre urinário (UFC) e a determinação do cortisol (sérico ou salivar) entre 23 horas e meia-noite $(4,5)$. Todos estão sujeitos a resultados falso-positivos e falso-negativos, e nenhum isoladamente permite uma distinção plena entre indivíduos com SC e aqueles com obesidade ou os chamados estados de pseudoCushing (por exemplo: ansiedade crônica, depressão e alcoolismo) (6-9).

A SC não ACTH-dependente, resultante sobretudo de tumores adrenais, é aquela cujos diagnóstico e tratamento geralmente são os menos complicados. O ACTH tipicamente está suprimido e a imagem tumoral é facilmente detectável pelo tomografia computadorizada $(2,10,11)$. A maior dificuldade reside na distinção entre a DC e a SAE. Mesmo em serviços de referência, a despeito da disponibilidade de recursos diagnósticos ilimitados, a fonte do excesso de ACTH pode permanecer obscura (3). As razões para essas dificuldades são diversas e incluem o tamanho diminuto dos corticotropinomas e de muitos tumores carcinóides brônquicos secretores de ACTH, os incidentalomas hipofisários e os casos de hipercortisolismo cíclico. Além disso, os achados clínicos e laboratoriais podem ser idênticos em pacientes com DC ou com tumores carcinóides secretores de ACTH (12-14).

$\mathrm{Na}$ diferenciação entre $\mathrm{DC}$ e SAE, os exames mais usados são o teste de supressão com doses altas de DMS (HDDST), o teste de estímulo com o hormônio liberador da corticotrofina $(\mathrm{CRH})$ ou a desmopressina (1-deamino-8-D-arginina vasopressina ou DDAVP), os estudos de imagem e o cateterismo bilateral do seio petroso inferior (CBSPI). Supressão do cortisol sérico ou do UFC > 50\% no HDDST, bem como in- cremento do ACTH $\geq 35 \%$ e/ou aumento do cortisol $\geq 20 \%$ após estímulo com CRH ovino ou DDAVP, são indicativos da doença de Cushing (15-18). Contudo, exceções a essa regra não são raras, principalmente com o HDDST (7). O ACTH plasmático tipicamente encontra-se normal ou elevado na DC e elevado na SAE, mas há grande superposição nos níveis desse hormônio (18).

A ressonância magnética permite a visualização de cerca de $60 \%$ dos corticotropinomas, cujo diâmetro médio é de 5,6 mm (16). O CBSPI tem sido considerado o exame mais acurado na distinção entre DC e SAE $(8,10)$. Nos estudos iniciais, observou-se uma acurácia diagnóstica de $100 \%(19,20)$. No entanto, posteriormente foram relatados casos de resultados falso-positivos e falso-negativos (21-23). Além disso, o CBSPI é um procedimento invasivo que requer um radiologista habilidoso e experiente com o método, e pode raramente se acompanhar de complicações neurológicas (24) ou tromboembólicas (25). O CBSPI, que nem sempre é conseguido devido a dificuldades técnicas, tem sua maior indicação nos casos em que a avaliação hormonal e os exames de imagem são discordantes $(1,8,10)$.

O presente estudo tem como objetivo principal analisar as características clínico-laboratoriais de 73 pacientes com síndrome de Cushing endógena, atendidos em dois serviços brasileiros de endocrinologia.

\section{PACIENTES E MÉTODOS}

\section{Pacientes}

Estudamos retrospectivamente 73 pacientes com diagnóstico de síndrome de Cushing endógena, atendidos no Serviço de Endocrinologia do Hospital das Clínicas da UFPE e no Centro de Diabetes e Endocrinologia de Pernambuco, em Recife, no período de 1994 a 2005.

\section{Desenho do estudo e ensaios}

Para confirmação do hipercortisolismo foram utilizadas as dosagem do cortisol sérico às 8 horas da manhã, cortisol livre urinário, cortisol sérico à meia-noite e cortisol salivar à meianoite, bem como os testes de supressão com doses baixas de dexametasona (LDDST). Estes últimos consistiram na dosagem do cortisol sérico às 8 horas após a administração oral de $1 \mathrm{mg}$ de dexametasona (DMS) às 23 horas da noite anterior e após a ingestão de $0,5 \mathrm{mg}$ de DMS a cada 6 horas por 48 horas (cortisol dosado exatamente 6 horas após a última tomada da DMS). Na interpretação dos LDDST, utilizamos como ponto de corte o valor de $1,8 \mu \mathrm{g} / \mathrm{dL}$, conforme sugerido por Wood e cols. (26). O cortisol salivar foi colhido na própria residência dos pacientes, enquanto que a coleta do cortisol sérico à meia-noite (CsMN) acon- 
teceu em ambiente hospitalar, após 2 dias de internamento. $\mathrm{O}$ paciente era acordado à meia-noite e a amostra sangüínea, retirada dentro de 5 minutos. Valores do CsMN superiores a $7,5 \mu \mathrm{g} / \mathrm{dL}$ foram considerados elevados (27).

Para determinação da etiologia do hipercortisolismo, dosou-se inicialmente o ACTH entre 8 e 9 horas da manhã, em duas ocasiões. Nos pacientes com supressão do ACTH, foi realizada tomografia computadorizada (TC) do abdome na investigação de tumores adrenais. Aqueles com níveis normais ou elevados de ACTH submeteram-se a dois testes dinâmicos: o teste de supressão com doses altas de DMS (HDDST) e o teste de estímulo com CRH ou DDAVP. No HDDST, o cortisol sérico (CS) às 8 horas foi dosado após supressão noturna com $8 \mathrm{mg}$ de DMS (administrada às 23 horas da noite anterior). Supressão maior de $50 \%$ no CS foi considerada indicativa de fonte hipofisária (doença de Cushing) (3-6). No teste de estímulo com CRH ou DDAVP, o ACTH foi dosado no estado basal (-15 e 0 minutos) e após a administração endovenosa (EV) de $100 \mu \mathrm{g}$ de CRH ovino ou $10 \mu \mathrm{g}$ de DDAVP $(15,30,45,60,90 \mathrm{e}$ 120 minutos). Um incremento nos níveis do ACTH $\geq 35 \%$ após CRH $(14,28)$ ou DDAVP $(14,29)$, em relação aos valores basais, foi considerado como indicativo de doença de Cushing. Não avaliamos a resposta do cortisol ao CRH ou DDAVP, considerando ser aquela do ACTH mais sensível e específica $(3,10,15,17)$.

$\mathrm{O}$ cateterismo bilateral do seio petroso inferior foi realizado em pacientes com síndrome de Cushing ACTHdependente e resultados discordantes entre os testes dinâmicos e os achados dos exames de imagem. O ACTH foi dosado no estado basal, bem como 2, 5, 10 e 15 minutos após a administração de $100 \mu \mathrm{g}$ de CRH ovino ou $10 \mu \mathrm{g}$ de DDAVP. Como previamente estabelecido, um gradiente de ACTH centro/periferia $\geq 2$ (basal) ou $\geq 3$ (após estímulo) foi considerado indicativo de doença de Cushing, enquanto valores menores foram interpretados como característicos da SAE $(2,17-21)$.
Ressonância magnética de crânio para estudo da sela túrcica foi realizada nos pacientes com síndrome de Cushing ACTH-dependente. Os pacientes com suspeita de SAE foram submetidos à TC de tórax e abdome.

\section{Ensaios}

O cortisol sérico e o cortisol livre urinário foram dosados por radioimunoensaio (RIE) ou quimioluminescência, o cortisol salivar por RIE, e o ACTH por RIE ou ensaio imunorradiométrico.

\section{Análise estatística}

Para a análise comparativa das variáveis quantitativas foi aplicado o teste de Análise de Variância (ANOVA). Para testar os contrastes, foi aplicado o teste de Levene para verificar a suposição de homogeneidade das variâncias. Assim, quando esta foi constatada, utilizou-se o teste de Tukey e, quando não, o teste de Tamhane. Para análise das variáveis qualitativas foi aplicado o teste Qui-quadrado ou, quando necessário, o teste exato de Fisher. Os resultados foram apresentados através dos valores de média ( \pm desvio-padrão) ou como proporções. Foi considerado estatisticamente significativo um valor de $\mathrm{p}<0,05$

\section{RESULTADOS}

\section{População de pacientes e características clínico-laboratoriais}

Um total de 73 pacientes foram avaliados, dos quais 46 (63\%) tinham doença de Cushing (DC), 21 $(28,7 \%)$ tumores adrenais e $6(8,2 \%)$, síndrome do ACTH ectópico (SAE) (tabela 1). Esta última resultou de 5 tumores carcinóides brônquicos e um carcinóide tímico. Houve predomínio do sexo feminino nos casos de DC $(74 \%)$ e tumores adrenais $(62 \%)$. Em contraste,

Tabela 1. Características dos pacientes de acordo com etiologia da Síndrome de Cushing.

\begin{tabular}{|c|c|c|c|}
\hline & Sexo (F/M) & $\begin{array}{c}\text { Idade (em anos) ao } \\
\text { diagnóstico } \\
\text { (média } \pm \text { DP) }\end{array}$ & $\begin{array}{c}\text { Tempo (em meses) } \\
\text { entre primeiros } \\
\text { sintomas e } \\
\text { diagnóstico } \\
\text { (média } \pm \text { DP) }\end{array}$ \\
\hline Doença de Cushing $(n=46)$ & $34 / 12$ & $14-52(36,9 \pm 7,9)$ & $12-37(25,0 \pm 5,2)$ \\
\hline $\operatorname{SAE}(n=6)$ & $2 / 5$ & $30-52(39,8 \pm 7,6)$ & $8-18(13,3 \pm 3,8)$ \\
\hline Adenoma adrenal $(n=15)$ & $10 / 5$ & $27-43(34,8 \pm 4,0)$ & $17-28(24,7 \pm 3,3)$ \\
\hline Carcinoma adrenal $(n=6)$ & $2 / 5$ & $3-48(35,0 \pm 16,3)$ & $14-24(18,2 \pm 3,8)$ \\
\hline p-valor & - & 0,603 & $<0,001$ \\
\hline \multirow[t]{4}{*}{ Diferenças significativas } & - & - & DC vs. CAA \\
\hline & & & DC vs. SAE \\
\hline & & & CAA vs. AA \\
\hline & & & AA vs. SAE \\
\hline
\end{tabular}

adenoma adrenal; $F=$ feminino; $M=$ masculino; $D P=$ desvio-padrão 
$66,6 \%$ dos pacientes com SAE eram do sexo masculino. A freqüência de manifestações clássicas do hipercortisolismo (ganho de peso/obesidade, face de "lua cheia", giba de búfalo, equimoses, adelgaçamento da pele e estrias violáceas) foi similar, independentemente de sua etiologia (tabela 2). O tempo passado entre o início dos primeiros sintomas do hipercortisolismo e o diagnóstico da síndrome de Cushing foi menor em pacientes com SAE e carcinomas adrenais do que naqueles com DC ou adenoma adrenal (tabela 1 ).

Diabetes mellitus [glicemia de jejum $(\mathrm{GJ}) \geq 126$ $\mathrm{mg} / \mathrm{dL}$ ] foi detectado em $30,1 \%$ dos casos, enquanto glicemia de jejum alterada (GJ entre 100 e $125 \mathrm{mg} / \mathrm{dL}$ ) ocorreu em 19,2\%. Hipertensão arterial (pressão arterial $>140 / 90 \mathrm{mmHg}$ ) se fez presente em $50,7 \%$. A prevalência desses distúrbios foi similar, a despeito da etiologia da síndrome de Cushing. Em contrapartida, hipocalemia $\left(\mathrm{K}^{+}\right.$sérico $\left.<3 \mathrm{mEq} / \mathrm{L}\right)$ foi significativamente mais freqüente nos casos de SAE (100\%) do que nos indivíduos com doença de Cushing (10,8\%) ou tumores adrenais $(9,5 \%)(\mathrm{p}<0,001)$ (tabelas 1 e 2$)$.

\section{Diagnóstico}

$\mathrm{Na}$ tabela 3 está resumido o comportamento dos testes diagnósticos. O cortisol sérico às 8 horas foi normal em 7 pacientes $(9,6 \%), 5$ com DC e 2 com adenomas adrenais. Perda da ritmicidade circadiana, evidenciada por elevação do cortisol (sérico ou salivar) à meia-noite, se fez presente em todos os pacientes em que foi avaliada. Entre 26 pacientes submetidos à dosagem do cortisol salivar à meianoite, os níveis do hormônio variaram de 12,6 a 26,7 $\mathrm{nmol} / \mathrm{L}$ (média de 18,2 $\pm 6,1$ ). O UFC foi avaliado em 52 pacientes $(71,2 \%)$, mostrando-se elevado em 46 deles $(88,5 \%)$ mas normal em 6 casos de DC. Os níveis médios do UFC mostraram-se significativamente mais elevados $(\mathrm{p}<0,001)$ nos pacientes com SAE $(696,3 \mu \mathrm{g} / 24 \mathrm{~h})$ do que naqueles com DC $(364,7 \mu \mathrm{g} / 24 \mathrm{~h})$ ou tumores adrenais $(424,6 \mu \mathrm{g} / 24 \mathrm{~h})$. Os testes de supressão com doses baixas de DMS (1 mg overnight e $2 \mathrm{mg} / 2$ dias) foram realizados em praticamente todos os pacientes. No primeiro teste, valores do cortisol sérico entre 2 e $5 \mu \mathrm{g} / \mathrm{dL}$, 5,1 e $10 \mu \mathrm{g} / \mathrm{dL}$, e > $10 \mu \mathrm{g} / \mathrm{dL}$ foram observados em, respectivamente, $5,5 \%, 20,5 \%$ e $74 \%$ dos pacientes. No segundo teste, os percentuais correspondentes foram $13,8,29,3 \%$ e $56,9 \%$, respectivamente (tabela 3 ).

\section{Diagnóstico diferencial}

Dosou-se o ACTH em todos os pacientes entre 8 e 9 horas da manhã e seus níveis $(\mathrm{em} \mathrm{pg} / \mathrm{mL}$ ) foram de 2,5-9,8 (média,7,4 $\pm 2,1), 18-320(88,1 \pm 53,1)$ e 175-1.820 (média 516,5 $\pm 654,1$ ) nos pacientes com tumores adrenais (TA), DC e SAE, respectivamente.
Os valores do ACTH revelaram-se suprimidos $(<10$ $\mathrm{pg} / \mathrm{mL}$ ) em $100 \%$ dos pacientes com TA, normais (em $37 \%$ ) ou elevados (em 63\%) na DC, e invariavelmente elevados na SAE. Houve superposição quando comparados os pacientes com DC ou SAE (figura 1), porém os valores médios do ACTH foram significativamente mais elevados na SAE $(\mathrm{p}<0,001)$.

O ACTH foi dosado por RIE em 21 pacientes (4 com tumores adrenais, 15 com doença de Cushing e 2 com SAE) e por ensaio imunorradiométrico nos demais (figura 1 ).

Os testes do CRH $(n=17)$ ou DDAVP $(n=26)$ foram realizados em 43 pacientes com síndrome de Cushing ACTH-dependente. Entre 37 pacientes com DC, $32(86,4 \%)$ demonstraram uma resposta positiva do ACTH (incremento $\geq 35 \%$ ), o mesmo acontecendo em $1(16,6 \%)$ paciente com SAE. Incremento do $\mathrm{ACTH} \geq 50 \%$ em relação aos níveis basais apenas ocorreu na doença de Cushing (sensibilidade de 70,2\% e especificidade de $100 \%$ ) (tabela 4 ).

No teste de supressão com doses altas de dexametasona (HDDST), supressão do cortisol sérico $>50 \%$, em relação ao valor basal, foi vista em 36/46 $(78,2 \%)$ pacientes com DC e em 2/6 (33,3\%) daqueles com SAE. No entanto, supressão do CS > $80 \%$ apenas foi observada na DC (presente em $48 \%$ dos casos) (tabela 4). Também foi exclusiva da DC a combinação de resposta do ACTH ao CRH ou DDAVP (incremento $\geq 35 \%$ ), e supressão do CS > $50 \%$ no HDDST, achado verificado em 24/37 $(64,9 \%)$ pacientes (tabela 4$)$.

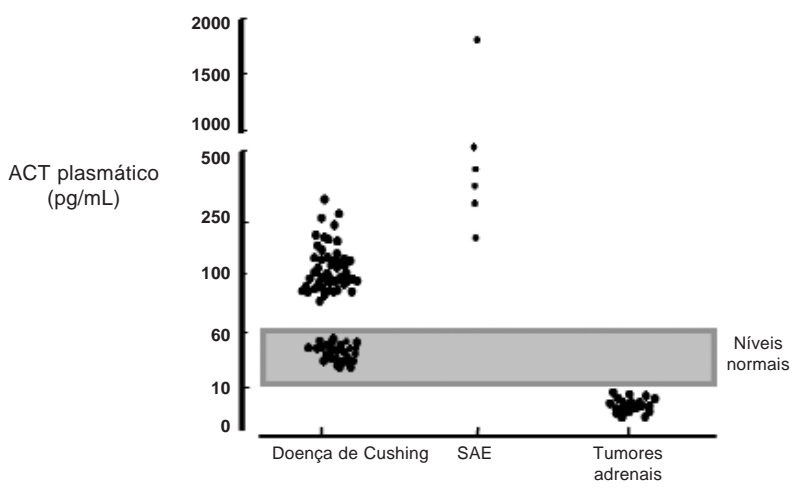

Figura 1. Níveis plasmáticos do ACTH $(e m \mathrm{pg} / \mathrm{mL}$ ) de acordo com a etiologia da síndrome de Cushing: 2,5-9,8 (média de $7,4 \pm 2,1$ ) nos tumores adrenais, 18-320 (média de 88,1 \pm 53,1 ) na doença de Cushing e 175-1.820 (média de 516,5 \pm $654,1)$ na síndrome do ACTH ectópico (SAE).

Obs: O ACTH foi dosado por RIE em 21 pacientes $(4 \mathrm{com}$ tumores adrenais, $15 \mathrm{com}$ doença de Cushing e $2 \mathrm{com}$ SAE) e por ensaio imunorradiométrico nos demais. 
Tabela 2. Manifestações clínico-laboratoriais de acordo com etiologia da Síndrome de Cushing.

\begin{tabular}{|c|c|c|c|c|c|c|}
\hline Sintomas/sinais & $\begin{array}{c}\text { Doença de } \\
\text { Cushing }\end{array}$ & SAE & p-valor* & $\begin{array}{c}\text { Adenoma } \\
\text { adrenal }\end{array}$ & $\begin{array}{c}\text { CA } \\
\text { adrenal }\end{array}$ & p-valor** \\
\hline $\begin{array}{l}\text { Ganho de peso / } \\
\text { obesidade }\end{array}$ & $\begin{array}{c}42 / 46 \\
(91,3 \%)\end{array}$ & $\begin{array}{c}4 / 6 \\
(66,7 \%)\end{array}$ & 0,14 & $\begin{array}{c}15 / 15 \\
(100 \%)\end{array}$ & $\begin{array}{c}5 / 6 \\
(83,3 \%)\end{array}$ & 0,28 \\
\hline Fraqueza & $\begin{array}{c}28 / 46 \\
(60,8 \%)\end{array}$ & $\begin{array}{c}5 / 6 \\
(83,3 \%)\end{array}$ & 0,39 & $\begin{array}{c}10 / 15 \\
(66,6 \%)\end{array}$ & $\begin{array}{c}6 / 6 \\
(100 \%)\end{array}$ & 0,62 \\
\hline Hipertensão & $\begin{array}{c}32 / 46 \\
(69,5 \%)\end{array}$ & $\begin{array}{c}4 / 6 \\
(66,7 \%)\end{array}$ & 1,00 & $\begin{array}{c}7 / 15 \\
(46,6 \%)\end{array}$ & $\begin{array}{c}4 / 6 \\
(66,7 \%)\end{array}$ & 0,63 \\
\hline $\begin{array}{l}\text { Face de } \\
\text { "Iua cheia" }\end{array}$ & $\begin{array}{c}37 / 46 \\
(80,4 \%)\end{array}$ & $\begin{array}{c}3 / 6 \\
(50 \%)\end{array}$ & 0,12 & $\begin{array}{c}13 / 15 \\
(86,6 \%)\end{array}$ & $\begin{array}{c}4 / 6 \\
(66,7 \%)\end{array}$ & 0,54 \\
\hline Giba de búfalo & $\begin{array}{c}26 / 46 \\
(56,5 \%)\end{array}$ & $\begin{array}{c}2 / 6 \\
(33,3 \%)\end{array}$ & 0,39 & $6 / 15(40 \%)$ & $3 / 6(50 \%)$ & 1,00 \\
\hline $\begin{array}{l}\text { Adelgaçamento } \\
\text { da pele }\end{array}$ & $\begin{array}{l}29 / 46 \\
(63 \%)\end{array}$ & $\begin{array}{c}2 / 6 \\
(33,3 \%)\end{array}$ & 0,21 & $\begin{array}{c}6 / 15 \\
(40 \%)\end{array}$ & $\begin{array}{c}2 / 6 \\
(33,3 \%)\end{array}$ & 1,00 \\
\hline Hirsutismo & $\begin{array}{l}17 / 34 \\
(50 \%)\end{array}$ & $\begin{array}{c}1 / 2 \\
(50 \%)\end{array}$ & 1,00 & $\begin{array}{c}4 / 10 \\
(40 \%)\end{array}$ & $\begin{array}{c}3 / 3 \\
(100 \%)\end{array}$ & 0,19 \\
\hline Acne & $\begin{array}{c}16 / 46 \\
(34,7 \%)\end{array}$ & $\begin{array}{c}3 / 6 \\
(50 \%)\end{array}$ & 0,65 & $\begin{array}{c}4 / 15 \\
(26,6 \%)\end{array}$ & $\begin{array}{c}3 / 6 \\
(50 \%)\end{array}$ & 0,35 \\
\hline Equimoses & $\begin{array}{c}32 / 46 \\
(69,5 \%)\end{array}$ & $\begin{array}{c}3 / 6 \\
(50 \%)\end{array}$ & 0,38 & $\begin{array}{c}10 / 15 \\
(66,6 \%)\end{array}$ & $\begin{array}{c}2 / 6 \\
(33,3 \%)\end{array}$ & 0,33 \\
\hline Pletora facial & $\begin{array}{l}35 / 46 \\
(76 \%)\end{array}$ & $\begin{array}{c}4 / 6 \\
(66,7 \%)\end{array}$ & 0,62 & $\begin{array}{c}10 / 15 \\
(66,6 \%)\end{array}$ & $\begin{array}{c}2 / 6 \\
(33,3 \%)\end{array}$ & 0,33 \\
\hline $\begin{array}{l}\text { Hiperpigmentação } \\
\text { cutânea }\end{array}$ & $\begin{array}{c}3 / 46 \\
(6,5 \%)\end{array}$ & $\begin{array}{c}2 / 6 \\
(33,3 \%)\end{array}$ & 0,09 & $\begin{array}{l}0 / 0 \\
(0 \%)\end{array}$ & $\begin{array}{c}0 / 0 \\
(0 \%)\end{array}$ & - \\
\hline Estrias violáceas & $\begin{array}{l}35 / 46 \\
(76 \%)\end{array}$ & $\begin{array}{c}3 / 6 \\
(50 \%)\end{array}$ & 0,32 & $\begin{array}{l}12 / 15 \\
(80 \%)\end{array}$ & $\begin{array}{c}2 / 6 \\
(33,3 \%)\end{array}$ & 0,11 \\
\hline Edema de MMII & $\begin{array}{c}15 / 46 \\
(32,6 \%)\end{array}$ & $\begin{array}{c}2 / 6 \\
(33,3 \%)\end{array}$ & 1,00 & $\begin{array}{c}5 / 15 \\
(33,3 \%)\end{array}$ & $\begin{array}{c}1 / 6 \\
(16,6 \%)\end{array}$ & 0,62 \\
\hline $\begin{array}{l}\text { Depressão e } \\
\text { outros distúrbios } \\
\text { psiquiátricos }\end{array}$ & $\begin{array}{c}11 / 46 \\
(23,9 \%)\end{array}$ & $\begin{array}{c}2 / 6 \\
(33,3 \%)\end{array}$ & 0,63 & $\begin{array}{c}4 / 15 \\
(26,6 \%)\end{array}$ & $\begin{array}{c}1 / 6 \\
(16,6 \%)\end{array}$ & 1,00 \\
\hline $\begin{array}{l}\text { Alterações } \\
\text { menstruais }\end{array}$ & $\begin{array}{c}24 / 34 \\
(70,5 \%)\end{array}$ & $\begin{array}{c}1 / 2 \\
(50 \%)\end{array}$ & 0,52 & $\begin{array}{c}7 / 10 \\
(70 \%)\end{array}$ & $\begin{array}{c}3 / 3 \\
(100 \%)\end{array}$ & 0,53 \\
\hline $\begin{array}{l}\text { Redução da libido } \\
\text { (ambos os sexos) }\end{array}$ & $\begin{array}{c}32 / 46 \\
(69,5 \%)\end{array}$ & $\begin{array}{c}4 / 6 \\
(66,7 \%)\end{array}$ & 1,00 & $\begin{array}{c}10 / 15 \\
(66,6 \%)\end{array}$ & $\begin{array}{c}3 / 5 \\
(50 \%)\end{array}$ & 1,0 \\
\hline $\begin{array}{l}\text { Osteopenia ou } \\
\text { osteoporose }\end{array}$ & $\begin{array}{c}20 / 32 \\
(62,5 \%)\end{array}$ & $\begin{array}{c}3 / 4 \\
(75 \%)\end{array}$ & 1,00 & $\begin{array}{c}4 / 8 \\
(50 \%)\end{array}$ & $\begin{array}{c}2 / 4 \\
(50 \%)\end{array}$ & - \\
\hline Disfunção erétil & $\begin{array}{c}6 / 12 \\
(50 \%)\end{array}$ & $\begin{array}{c}2 / 4 \\
(50 \%)\end{array}$ & 1,00 & $\begin{array}{c}3 / 6 \\
(50 \%)\end{array}$ & $\begin{array}{c}2 / 3 \\
(66,6 \%)\end{array}$ & 1,00 \\
\hline $\begin{array}{l}\text { Diabetes mellitus } \\
\text { (glicemia de jejum } \\
\geq 126 \mathrm{mg} / \mathrm{dL} \text { ) }\end{array}$ & $\begin{array}{c}14 / 46 \\
(30,4 \%)\end{array}$ & $\begin{array}{c}3 / 6 \\
(50 \%)\end{array}$ & 0,38 & $\begin{array}{c}4 / 15 \\
(26,6 \%)\end{array}$ & $\begin{array}{c}2 / 6 \\
(33,3 \%)\end{array}$ & 1,00 \\
\hline $\begin{array}{l}\text { Glicemia de jejum } \\
\text { alterada ( } \geq 100 \mathrm{e} \\
<126 \mathrm{mg} / \mathrm{dL})\end{array}$ & $\begin{array}{c}10 / 46 \\
(21,7 \%)\end{array}$ & $\begin{array}{c}1 / 6 \\
(16,6 \%)\end{array}$ & 1,00 & $\begin{array}{c}2 / 15 \\
(13,3 \%)\end{array}$ & $\begin{array}{c}1 / 6 \\
(16,6 \%)\end{array}$ & 1,00 \\
\hline $\begin{array}{l}\text { Hipocalemia } \\
\left(\mathrm{K}^{+}<3 \mathrm{mEq} / \mathrm{L}\right)\end{array}$ & $\begin{array}{c}5 / 46 \\
(10,8 \%)\end{array}$ & $\begin{array}{c}6 / 6 \\
(100 \%)\end{array}$ & $<0,001$ & $\begin{array}{c}1 / 15 \\
(6,6 \%)\end{array}$ & $\begin{array}{c}1 / 6 \\
(16,6 \%)\end{array}$ & 0,50 \\
\hline
\end{tabular}

* Relativo à comparação entre pacientes com doença de Cushing ou SAE.

* Relativo à comparação entre pacientes com adenoma ou carcinoma adrenais.

$\mathrm{SAE}=$ síndrome do $\mathrm{ACTH}$ ectópico; $\mathrm{CA}=$ carcinoma; $\mathrm{MMII}=$ membros inferiores 
Tabela 3. Comportamento dos exames usados para o diagnóstico da síndrome de Cushing.

\begin{tabular}{|c|c|c|c|c|}
\hline Exame & $\begin{array}{l}\text { Valor de } \\
\text { referência }\end{array}$ & $\begin{array}{c}\mathbf{N}^{\circ} \text { de } \\
\text { pacientes }\end{array}$ & $\begin{array}{c}\text { Variação } \\
\text { (média } \pm \text { desvio-padrão) }\end{array}$ & $\begin{array}{c}\% \text { de } \\
\text { pacientes } \\
\text { com valores } \\
\text { elevados }\end{array}$ \\
\hline $\begin{array}{l}\text { Cortisol sérico às } \\
8: 00 \mathrm{~h}(\mu \mathrm{g} / \mathrm{dL})\end{array}$ & $5-25$ & 73 & $20,4-49,2(30,3 \pm 5,5)$ & $90,4 \%$ \\
\hline $\begin{array}{l}\text { CS à meia-noite } \\
(\mu \mathrm{g} / \mathrm{dL})\end{array}$ & $\leq 7,5$ & 35 & $11-34,8(19,3 \pm 5,3)$ & $100 \%$ \\
\hline $\begin{array}{l}\text { Cortisol salivar } \\
\text { à meia-noite } \\
\text { (nmol/L) }\end{array}$ & $\leq 8,5$ & 26 & $12,6-26,7(18,2 \pm 6,1)$ & $100 \%$ \\
\hline $\begin{array}{l}\text { CS no teste de } \\
\text { supressão noturna } \\
\text { com } 1 \mathrm{mg} \text { de DMS ( } \mu \mathrm{g} / \mathrm{dL})\end{array}$ & $\leq 1,8$ & 73 & $3,6-28,5(12,9 \pm 4,4)$ & $100 \%$ \\
\hline $\begin{array}{l}\text { CS após supressão } \\
\text { com } 2 \mathrm{mg} \text { de } \mathrm{DMS} / 2 \text { dias } \\
(\mu \mathrm{g} / \mathrm{dL})\end{array}$ & $\leq 1,8$ & 72 & $2,0-18,2(6,9 \pm 3,8)$ & $100 \%$ \\
\hline $\begin{array}{l}\text { Cortisol livre } \\
\text { urinário ( } \mu \mathrm{g} / 24 \mathrm{~h})\end{array}$ & $10-90$ & 52 & $62-1760(390,7 \pm 336,4)$ & $88,5 \%$ \\
\hline
\end{tabular}

Tabela 4. Desempenho dos testes usados para o diagnóstico diferencial da síndrome de Cushing ACTH-dependente.

\begin{tabular}{|c|c|c|c|c|c|}
\hline \multirow[t]{2}{*}{ TESTE (Critérios adotados) } & \multirow[t]{2}{*}{ Sensibilidade } & \multirow[t]{2}{*}{ Especificidade } & \multirow{2}{*}{$\begin{array}{c}\text { Acurácia } \\
\text { diagnóstica }\end{array}$} & \multicolumn{2}{|c|}{ Valor preditivo } \\
\hline & & & & Positivo & Negativo \\
\hline \multicolumn{6}{|c|}{ TESTE DE ESTÍMULO COM CRH OVINO OU DDAVP } \\
\hline Incremento do $\mathrm{ACTH} \geq 35 \%$ & $81,0 \%$ & $83,3 \%$ & $81,4 \%$ & $96,8 \%$ & $41,7 \%$ \\
\hline Incremento do ACTH $\geq 50 \%$ & $70,3 \%$ & $100,0 \%$ & $74,4 \%$ & $100,0 \%$ & $35,3 \%$ \\
\hline \multicolumn{6}{|l|}{ HDDST (COM 8 MG OVERNIGHT) } \\
\hline Supressão do CS > 50\% & $78,2 \%$ & $66,7 \%$ & $77,8 \%$ & $95,0 \%$ & $28,6 \%$ \\
\hline Supressão do CS > 80\% & $47,8 \%$ & $100,0 \%$ & $53,8 \%$ & $100,0 \%$ & $20,0 \%$ \\
\hline \multicolumn{6}{|c|}{ HDDST (COM 8 MG OVERNIGHT) + TESTE DE ESTÍMULO COM CRH OVINO OU DDAVP } \\
\hline Supressão do CS > 50\% no & & & & & \\
\hline $\begin{array}{l}\text { HDDST+ Pico do ACTH } \geq 35 \\
\text { pós-CRH ou DDAVP } \\
\text { CBSPI }\end{array}$ & $64,9 \%$ & $100,0 \%$ & $82,4 \%$ & $100,0 \%$ & $74,0 \%$ \\
\hline $\begin{array}{l}\text { Gradiente de ACTH } \\
\text { centro:periferia basal } \geq 2\end{array}$ & $100,0 \%$ & $100,0 \%$ & $100,0 \%$ & $100,0 \%$ & $100,0 \%$ \\
\hline $\begin{array}{l}\text { Gradiente de ACTH } \\
\text { centro:periferia } \geq 3 \text { após } \mathrm{CRH} \\
\text { ou DDAVP }\end{array}$ & $90 \%$ & $100,0 \%$ & $93,8 \%$ & $100,0 \%$ & $85,7 \%$ \\
\hline
\end{tabular}

$\mathrm{CBSPI}=$ Cateterismo bilateral do seio petroso inferior; HDDST= teste de supressão com doses altas de dexametasona; $\mathrm{CS}=$ cortisol sérico

\section{Estudos de imagem}

A ressonância magnética $(\mathrm{RM})$ da hipófise foi realizada nos 52 pacientes com síndrome de Cushing ACTHdependente. Entre os 46 pacientes com DC, mostrou a presença de 9 macroadenomas (19,5\%). Além disso, visualizou a lesão em 22 dos $37(59,4 \%)$ portadores de microadenomas. Em um paciente com um tumor carcinóide brônquico, a RM mostrou uma imagem sugestiva de um microadenoma hipofisário com $5 \mathrm{~mm}$.
A tomografia computadorizada (TC) possibilitou a identificação de cinco tumores carcinóides brônquicos e um carcinóide tímico. A TC abdominal foi realizada nos pacientes com síndrome de Cushing não ACTH-dependente e visualizou 100\% dos adenomas e carcinomas adrenais. Nos primeiros, o tamanho da lesão, no seu maior diâmetro, variou de 1,5 a $4 \mathrm{~cm}$ (média de 2,8 $\pm 0,7 \mathrm{~cm}$ ). Os carcinomas mediram de 6 a 13,5 cm (média de 9,1 $\pm 3,0 \mathrm{~cm}$ ). 


\section{Cateterismo bilateral do seio petroso inferior (CBSPI)}

CBSPI foi realizado em 10 pacientes com síndrome de Cushing ACTH-dependente que posteriormente tiveram o diagnóstico confirmado de doença de Cushing (DC). Um gradiente centro-periferia de ACTH maior ou igual a 3 após estímulo com CRH ( 3 pacientes) ou DDAVP ( 7 pacientes) teve sensibilidade de $90 \%$ e especificidade de $100 \%$, uma vez que em um paciente o referido gradiente foi de 2,4 (tabela 4).

\section{DISCUSSÃO}

Este estudo analisou as características clínicas e laboratoriais de um grupo de 73 pacientes com síndrome de Cushing endógena e demonstrou as inerentes dificuldades no diagnóstico dessa patologia. Observamos que a doença de Cushing respondeu por 63\% dos casos, os tumores adrenais por $28,7 \%$ e a síndrome do ACTH ectópico (SAE) por 8,2\%. Esses percentuais são comparáveis aos descritos em um estudo multicêntrico italiano com 426 pacientes $(67 \%, 29,1 \%$ e $5,8 \%$, respectivamente) (30). Entre nossos pacientes com SAE, $5(83,3 \%)$ tinham um tumor carcinóide brônquico. Esse dado é consistente com os descritos por outros autores que mostraram ser os carcinóides brônquicos a principal etiologia de secreção ectópica de ACTH $(12,13)$.

Com relação aos exames de rastreamento ou para confirmação do hipercortisolismo, evidenciamos que, usando o ponto de corte de $1,8 \mu \mathrm{g} / \mathrm{dL}$, nenhum paciente apresentou supressão do cortisol sérico durante os testes de supressão com baixas doses de dexametasona (LDDST). Resultados comparáveis foram encontrados em estudos do Reino Unido que usaram o mesmo protocolo e mostraram uma sensibilidade de $98-100 \%$ nos LDDST para confirmação do hipercortisolismo $(12,26,31)$. Em estudo anterior, mostramos que o LDDST com $2 \mathrm{mg} / 48$ horas permitiu uma clara distinção entre pacientes obesos e aqueles com síndrome de Cushing (32). Seis pacientes (11,5\%) com síndrome de Cushing no presente estudo apresentaram-se com níveis normais do cortisol livre urinário (UFC). Esse percentual situou-se entre 5\% e $15 \%$ na experiência da maioria dos autores (2-8), mas na série de Lin e cols. (33) atingiu $28 \%$. Entre 15 pacientes com síndrome de Cushing confirmada, os LDDST e o cortisol sérico à meia-noite tiveram sensibilidade de $100 \%$ (34). No entanto, em 2 pacientes (13,4\%), o UFC foi normal (34). Inadequação na coleta da amostra urinária de 24 horas e hipercortisolismo intermitente estão entre os principais determinantes de síndrome de Cushing com níveis normais de UFC $(1,5)$. Observamos, também, que o cortisol salivar à meia-noite mostrou-se elevado em todos os pacientes em que foi testado (tabela 3), confirmando seu importante papel como exame de rastreamento para síndrome de Cushing, bem salientado em estudos recentes $(35,36)$.

No presente estudo, os níveis de ACTH revelaram-se suprimidos $(<10 \mathrm{pg} / \mathrm{mL})$ em $100 \%$ dos casos de tumores adrenais, normais $(30,5 \%)$ ou elevados $(69,5 \%)$ na DC, e elevados em $100 \%$ dos indivíduos com SAE. Os níveis médios de ACTH, bem como os do UFC, mostraram-se significativamente mais elevados na SAE do que na DC, ratificando assim o achado de outros estudos $(30,37)$. Entre os pacientes com doença de Cushing avaliados por Invitti e cols. (30), 56\% tinham o ACTH plasmático normal. Nas séries de Isidori e cols. (12) e Loli e cols. (14), também se constatou elevação do ACTH em todos os casos de SAE. No entanto, em outros dois estudos $(13,30), 17-32 \%$ dos pacientes com SAE tinham valores normais do ACTH plasmático.

Supressão $>50 \%$ do cortisol sérico (CS) no teste de supressão com doses altas de dexametasona (HDDST) foi observada em $78,2 \%$ de nossos pacientes com DC, mas também ocorreu em um terço dos casos de SAE. Em três estudos recentes (12-14), supressão do cortisol > 50\% foi relatada em 10 a 33,3\% dos casos de SAE. Esses percentuais são condizentes com estudos anteriores que revelaram ter o HDDST com $8 \mathrm{mg}$ overnight sensibilidade e especificidade de $77-92 \%$ e $57-100 \%$, respectivamente (38-40). Por outro lado, entre os nossos pacientes, supressão > 80\% apenas ocorreu na DC (presente em $48 \%$ dos casos). Esses achados estão de acordo com os verificados no estudo multicêntrico italiano (30) em que supressão > 80\% no CS ou UFC teve especificidade de $100 \%$ para DC. Contudo, existem ao menos dois casos descritos de SAE em que a supressão do CS ou UFC excedeu 90\% durante o HDDST $(14,41)$.

Observamos que resposta positiva do ACTH (incremento $\geq 35 \%$ em relação ao basal) se fez presente em $81 \%$ dos pacientes com DC submetidos ao teste de estímulo com CRH ovino ou DDAVP. Resposta similar foi evidenciada em um paciente $(16,6 \%)$ com SAE. Em outros estudos, sensibilidade e especificidade do teste do CRH foram de $86-93 \%$ e $88-100 \%$, respectivamente, considerando a resposta do ACTH e cortisol $(1,6,10,15,28)$. Na série de Loli e cols. (14), entre 6 pacientes com carcinóides brônquicos ocultos secretores de $\mathrm{ACTH}$, a administração de $\mathrm{CRH}$ ovino não induziu nenhum aumento do ACTH e/ou cortisol em 4 pacientes $(66,6 \%)$. No entanto, um paciente teve um aumento de $38 \%$ no ACTH e um outro, incremento de 
$30 \%$ no cortisol. Resposta do cortisol e ACTH à administração de DDAVP ocorreu em $2 / 4$ pacientes (50\%) (14). Em estudo mais recente, apenas 9\% dos pacientes com SAE responderam à administração do CRH (13). Na nossa casuística, resposta a ambos CRH (ou DDAVP) e HDDST apenas ocorreu na DC (sensibilidade de $64,9 \%$ e especificidade de 100\%). Excepcionalmente, tal resposta é vista na SAE, tendo ocorrido em um paciente das séries de Isidori e cols. (12) e Ilias e cols. (13). Em contrapartida, ausência de resposta a ambos CRH e HDDST é bastante sugestiva de SAE, e tal comportamento foi verificado em 79\% dos pacientes avaliados por Ilias e cols. (13). Incremento do ACTH > 50\% após estímulo com CRH ou DDAVP também foi exclusivo da DC na nossa casuística (presente em 70\% dos casos). No estudo multicêntrico italiano, incremento do ACTH > 50\% pósCRH também teve $100 \%$ de especificidade para DC, com sensibilidade de $85 \%$ (30). No entanto, em outras duas séries $(16,42)$, esse achado teve sensibilidade e especificidade de $86 \%$ e 90-95\%, respectivamente.

Classicamente, hipocalemia é bem mais freqüente em pacientes com SAE do que naqueles com outras formas de síndrome de Cushing, em decorrência da maior intensidade do hipercortisolismo (1$3)$. Na nossa casuística, hipocalemia foi significativamente mais comum na SAE do que na DC (100\% vs. $11 \%, \mathrm{p}<0,001)$. Entre os pacientes com carcinóides brônquicos relatados por Loli e cols. (14), todos também apresentavam níveis séricos de $\mathrm{K}^{+}<3 \mathrm{mEq} / \mathrm{L}$. Contudo, quando analisados 130 casos de SAE, a prevalência de hipocalemia foi de aproximadamente 70\% $(12,13)$. Conforme mostrado na tabela 2 , a freqüência de manifestações clássicas da síndrome de Cushing foi similar em pacientes com DC e SAE.

A ressonância magnética $(\mathrm{RM})$ visualizou $100 \%$ dos macroadenomas e $63,5 \%$ dos microadenomas hipofisários. Um dos pacientes com SAE apresentou também uma imagem sugestiva de um microadenoma hipofisário. Na série de Ilias e cols. (13), 17 de 66 (26\%) dos pacientes com SAE tinham uma RM hipofisária anormal. Ademais, incidentalomas hipofisários e adrenais são encontrados, respectivamente, em 10\% e até $4 \%$ da população adulta geral $(1,18,43)$. Portanto, os exames de imagem isoladamente não permitem a definição da etiologia do hipercortisolismo.

Ao cateterismo bilateral do seio petroso inferior (CBSPI), um de nossos pacientes com doença de Cushing teve um gradiente centro:periferia de ACTH pós-DDAVP $<3$, sugestivo de SAE. Resultados falsonegativos e falso-positivos foram também relatados por outros autores $(22,23)$. Uma análise de 14 séries, totalizando 726 pacientes com doença de Cushing e 112 com SAE, revelou 41 resultados falso-negativos e 7 falso-positivos no CBSPI, conferindo sensibilidade e especificidade de $94 \%$ para esse exame (10).

Em resumo, nossos achados demonstraram que os aspectos clínicos foram similares nos pacientes com doença de Cushing, tumores adrenais ou tumores carcinóides secretores de ACTH e, portanto, sem valor na distinção entre essas condições. Entre os testes de rastreamento para o hipercortisolismo, a dosagem de cortisol livre urinário teve sensibilidade inferior à dos testes de supressão com doses baixas de dexametasona e a mensuração do cortisol à meia-noite (sérico ou salivar). Da mesma forma, observamos que os critérios clássicos do teste de supressão com doses altas de dexametasona (HDDST) e teste de estímulo com CRH ou DDAVP isoladamente apresentaram especificidade limitada na diferenciação entre a doença de Cushing e a síndrome do ACTH ectópico. No entanto, uma resposta positiva em ambos os testes revelou ter alta acurácia diagnóstica, o mesmo se aplicando para critérios mais rígidos de supressão do cortisol sérico (> 80\%) no HDDST e incremento do ACTH (> 50\%) após CRH ou DDAVP.

\section{REFERÊNCIAS}

1. Vilar L, Coelho CE. Diagnóstico e diagnóstico diferencial da síndrome de Cushing. In: Vilar L, Kater CE, Naves LA, et al. (eds). Endocrinologia Clínica. $3^{a}$ edição. Rio de Janeiro: Guanabara Koogan, 2006. pp. 397-417.

2. Newell-Price J, Bertagna X, Grossman AB, Nieman LK. Cushing's syndrome. Lancet 2006;367:1605-17.

3. Newell-Price J, Trainer P, Besser GM, Grossman A. The diagnosis and differential diagnosis of Cushing's syndrome and pseudo-Cushing's states. Endocr Rev 1998;19:647-72.

4. Crapo L. Cushing's syndrome: A review of diagnostic tests. Metabolism 1979;28:955-77.

5. Miller J, Crapo L. The biochemical diagnosis of hypercortisolism. Endocrinologist 1994;4:7-16.

6. Castro M, Moreira AC. Diagnóstico laboratorial da síndrome de Cushing. Arq Bras Endocrinol Metab 2002;46:97-105.

7. Findling JW, Raff H. Newer diagnostic techniques and problems in Cushing's disease. Endocrinol Metab Clin North Am 1999;28:191-210.

8. Arnaldi G, Angeli A, Atkinson AB, Bertagna X, Cavagnini F, Chrousos GP, et al. Diagnosis and complications of Cushing's syndrome: a consensus statement. J Clin Endocrinol Metab 2003;88:5593-602.

9. Findling JW, Raff H. Screening and diagnosis of Cushing's syndrome. Endocrinol Metab Clin North Am 2005;34:385-402.

10. Lindsay JR, Nieman LK. Differential diagnosis and imaging in Cushing's syndrome. Endocrinol Metab Clin North Am 2005;34:403-22.

11. Kirk LF Jr, Hash RB, Katner HP, Jones T. Cushing's disease: clinical manifestations and diagnostic evaluation. Am Fam Physician 2000;62:1119-27.

12. Isidori AM, Kaltsas GA, Pozza C, Frajese V, Newell-Price J, Reznek $\mathrm{RH}$ et al. The ectopic adrenocorticotropin syndrome: clinical features, diagnosis, management, and long-term follow-up. J Clin Endocrinol Metab 2006;91:371-7. 
13. Ilias I, Torpy DJ, Pacak K, Mullen N, Wesley RA, Nieman LK. Extensive clinical experience. Cushing's syndrome due to ectopic corticotropin secretion: twenty years' experience at the National Institutes of Health. J Clin Endocrinol Metab 2005;90:4955-62.

14. Loli P, Vignati F, Grossrubatscher E, Dalino P, Possa M, Zurleni $F$, et al. Management of occult adrenocorticotropinsecreting bronchial carcinoids: limits of endocrine testing and imaging techniques. J Clin Endocrinol Metab 2003;88:1029-35.

15. Morris DG, Grossman AB. Dynamic tests in the diagnosis and differential diagnosis of Cushing's syndrome. J Endocrinol Invest 2003;26(suppl. 7):64-73.

16. Kaye TB, Crapo L. The Cushing syndrome: an update on diagnostic tests. Ann Intern Med 1990;112:434-44.

17. Nieman LK. Diagnostic tests for Cushing's syndrome. Ann $\mathbf{N}$ Y Acad Sci 2002;970:112-8.

18. Vilar L, Naves L. Pitfalls in the diagnosis of Cushing's syndrome. Arq Bras Endocrinol Metab 2003:47(supl. 1):S255-9.

19. Oldfield EH, Doppman JL. Petrosal versus cavernous sinus sampling. J Neurosurg 1998;89:890-3.

20. Findling JW, Kehoe ME, Shaker JL, Raff H. Routine inferior petrosal sinus sampling in the differential diagnosis of adrenocorticotropin (ACTH)-dependent Cushing's syndrome: early recognition of the occult ectopic ACTH syndrome. $\mathbf{J}$ Clin Endocrinol Metab 1991;73:408-13.

21. Pecori Giraldi F, Invitti C, Cavagnini F. Inferior petrosal sinus sampling ten years down the road. J Endocrinol Invest 2000;23:325-7.

22. Swearingen B, Katznelson L, Miller K, Grinspoon S, Waltman A, Dorer DJ, et al. Diagnostic errors after inferior petrosal sinus sampling. J Clin Endocrinol Metab 2004;89:3752-63.

23. Yamamoto $Y$, Davis DH, Nippoldt TB, Young WF Jr, Huston J 3rd, Parisi JE. False-positive inferior petrosal sinus sampling in the diagnosis of Cushing's disease. J Neurosurg 1995:83:1087-91.

24. Miller DL, Doppman JL, Peterman SB, Nieman LK, Oldfield $\mathrm{EH}$, Chang R. Neurologic complications of petrosal sinus sampling. Radiology 1992;185:143-74

25. Blevins LS Jr, Clark RV, Owens DS. Thromboembolic complications after inferior petrosal sinus sampling in patients with Cushing's syndrome. Endocr Pract 1998;4:365-7.

26. Wood PJ, Barth JH, Freedman DB, Perry L, Sheridan B. Evidence for the low dose dexamethasone suppression test to screen for Cushing's syndrome-recommendations for a protocol for biochemistry laboratories. Ann Clin Biochem 1997;34:222-9.

27. Papanicolaou DA, Yanovski JA, Cutler GB, Chrousos GB, Nieman LK. A single midnight serum cortisol measurement distinguishes Cushing's syndrome from pseudo-Cushing states. J Clin Endocrinol Metab 1998:83:1163-7.

28. Nieman LK, Oldfield EH, Wesley R, Chrousos GP, Loriaux DL, Cutler GB Jr. A simplified morning ovine corticotropin-releasing hormone stimulation test for differential diagnosis of adrenocorticotropin-dependent Cushing's syndrome. J Clin Endocrinol Metab 1993;77:1308-12.

29. Newell-Price J, Perry L, Medbak S, Monson J, Savage M, Besser $\mathrm{M}$, et al. A combined test using desmopressin and corticotropin-releasing hormone in the differential diagnosis of Cushing's syndrome. J Clin Endocrinol Metab 1997:82:176-81.

30. Invitti C, Giraldi FP, de Martin M, Cavagnini F. Diagnosis and management of Cushing's syndrome: results of an Italian multicentre study. Study group of the Italian Society of Endocrinology on the pathophysiology of the hypothalamic-pituitary-adrenal axis. J Clin Endocrinol Metab 1999;84:440-8.
31. Newell-Price J, Trainer P, Perry L, Wass J, Grossman A, Besser M. A single sleeping midnight cortisol has $100 \%$ sensitivity for the diagnosis of Cushing's syndrome. Clin Endocrinol (Oxf) 1995;43:545-50.

32. Vilar L, Freitas MC, Canadas V, Moura E, Leal E, Teixeira L, et al. Comportamento dos testes de supressão com doses baixas de dexametasona (LDDST) na obesidade e na síndrome de Cushing. Arq Bras Endocrinol Metab 2002;46(supl. 1):S493.

33. Lin CL, Wu TJ, Machacek DA, Jiang NS, Kao PC. Urinary free cortisol and cortisone determined by high performance liquid chromatography in the diagnosis of Cushing's syndrome. $\mathbf{J}$ Clin Endocrinol Metab 1997;82:151-5.

34. Vilar L, Moura E, Campos R, Gusmão A, Leal E, Teixeira L, et al. Comparative efficacy of diagnostic tests to confirm Cushing's syndrome. Arq Bras Endocrinol Metab 2003;47(supl. 1):S348.

35. Yaneva M, Mosnier-Pudar H, Dugué MA, Grabar S, Fulla Y, Bertagna $X$. Midnight salivary cortisol for the initial diagnosis of Cushing's syndrome of various causes. J Clin Endocrinol Metab 2003;89:3345-51.

36. Putignano $P$, Toja $P$, Dubini A, Giraldi FP, Corsello SM, Cavagnini $F$. Midnight salivary cortisol versus urinary free and midnight serum cortisol as screening tests for Cushing's syndrome. J Clin Endocrinol Metab 2003;88:4153-7.

37. Isidori AM, Kaltsas GA, Grossman AB. Ectopic ACTH syndrome. Front Horm Res 2006;35:143-56.

38. Dichek HL, Nieman LK, Oldfield EH, Pass HI, Malley JD, Cutler Jr GB. A comparison of the standard high dose dexamethasone suppression test and the overnight 8-mg dexamethasone suppression test for the differential diagnosis of adrenocorticotropin-dependent Cushing's syndrome. J Clin Endocrinol Metab 1994;78:418-22.

39. Tyrrell JB, Findling JW, Aron DC, Fitzgerald PA, Forsham PH. An overnight high-dose dexamethasone suppression test for rapid differential diagnosis of Cushing's syndrome. Ann Intern Med 1986;104:180-6.

40. Bruno OD, Rossi MA, Contreras LN, Gomez RM, Galparsoro $\mathrm{G}$, Cazado E, et al. Nocturnal high-dose dexamethasone suppression test in the aetiological diagnosis of Cushing's syndrome. Acta Endocrinol (Copenh) 1985;109:158-62.

41. Findling JW, Raff $H$. Diagnosis and differential diagnosis of Cushing's syndrome. Endocrinol Metab Clin 2001;30:729-47.

42. Reimondo G, Paccotti $P$, Minetto M, Termine A, Stura G, Bergui $M$, et al. The corticotrophin-releasing hormone test is the most reliable noninvasive method to differentiate pituitary from ectopic ACTH secretion in Cushing's syndrome. Clin Endocrinol (Oxf) 2003;58;718-24.

43. Vilar L, Azevedo MF, Barisic G, Naves LA. Incidentalomas hipofisários. Arq Bras Endocrinol Metab 2005;49:651-6.

\section{Endereço para correspondência:}

Lucio Vilar

Rua Clovis Silveira Barros 84/1202

50050-270 Recife, PE

E-mail: Ivilar@gmail.com 